\title{
A BEM for the Propagation of Nonlinear Planar Free-surface Waves
}

\author{
V. Vinayan ${ }^{1}$, S. A. Kinnas ${ }^{2}$ \\ Ocean Engineering Group, The University of Texas at Austin \\ Civil, Architectural and Environmental Engineering Department \\ 1 University Station C1786, Austin, TX 78712
}

\begin{abstract}
A Boundary Element Method (BEM) model for the propagation of nonlinear free-surface waves is described and its application to the study of the hydrodynamic characteristics associated with the roll-motion of 2-D hull sections is presented. The roll-motion of the hull section is modeled as a mixed boundary value problem and solved using a higher-order (linear strength distribution) BEM coupled with a Mixed-Eulerian-Lagrangian (MEL) scheme for the time-dependent free-surface boundary conditions. Applications, that include the propagation of fifth-order Stokes waves and waves generated by a piston wave-maker, used to validate the BEM scheme prior to its application to the hull roll-motion are also described.
\end{abstract}

\section{Introduction}

In comparison to Finite Difference Methods (FDM), Finite Volume Methods (FVM) and other numerical techniques, the Boundary Element Method (BEM) can be used to model the free-surface with relative ease within the framework of potential theory. Potential-flow methods have been extensively used for the numerical solution of water waves and studies of wave-body interactions (see Longuet-Higgins and Cokelet [1976], Faltinsen [1977], Vinje and Brevig [1981], Lin et al. [1984], Dold and Peregrine [1984], Grosenbaugh and Yeung [1989], Grilli and Svendsen [1989] for 2-D applications, and Liu et al. [2001], Sung and Grilli [2005] for more recent high-order 3-D BEM applications). A detailed review of the application of BEM and other numerical methods for the computation of nonlinear free-surface flows and past research can be found in Yeung [1982], Tsai and Yue [1996].

The BEM model presented here forms an integral part of an overall research effort to model the unsteady, viscous flow associated with the roll-motion of 2-D hull-sections with or without bilge keels. The focus of the research is on obtaining the hydrodynamic added-mass and damping coefficients at different amplitudes and

\footnotetext{
${ }^{1}$ Doctoral Graduate Student, vvinayan@mail.utexas.edu (corresponding author)

${ }^{2}$ Professor, kinnas@mail.utexas.edu
} 
frequencies of roll-motion. Viscous effects, neglected in the potential-flow based BEM scheme, play a major role in damping hull roll-motions. A FVM based NavierStokes Solver (Kinnas et al. [2003], Yu et al. [2005]), developed independently, is used to capture the viscous effects combined with linear boundary conditions on the free-surface. In this context, the aim of the BEM model is to study the extent to which the linear free-surface boundary conditions are applicable.

It is important that any numerical model be validated against analytical or experimental results if available. This paper primarily focusses on the validation studies performed to verify the accuracy of the BEM model in propagating nonlinear free-surface waves. Two validation studies are performed : (1) The propagation of Stokes waves based on the fifth-order gravity wave theory of Fenton [1985]. In this case the numerical results are compared with the analytical wave profiles. (2) The waves generated by the sinusoidal motion of a piston wave-maker. The results obtained from the BEM model are compared with the numerical results of Lin [1984] in this case. Representative results for the roll-motion analysis of FPSO hull-sections are also presented.

\section{Mathematical Formulation}

An ideal fluid is assumed and the flow is described in terms of a velocity potential with the additional assumption of being irrotational. A Cartesian coordinate system, $\mathbf{x}=(x, y)$, fixed in space is chosen with the origin at the undisturbed water level. $x$ represents the horizontal direction and $y$ the vertical direction, positive upward. The flow, expressed in terms of the velocity potential $\varphi(\mathbf{x}, t)$, satisfies the Laplace equation

$$
\nabla^{2} \varphi(\mathbf{x}, t)=0 ; \quad \mathbf{x} \in \mathbf{\Omega}(t)
$$

where $t$ is the time and $\boldsymbol{\Omega}(t)$ represents the fluid domain. In general, the roll-motion analysis or the propagation of waves is formulated as an initial boundary-value problem (BVP) for the velocity potential $\varphi(\mathbf{x}, t)$ and solved with either Neumann or Dirichlet type conditions specified on the boundaries. In the next three sections, the initial and boundary conditions specific to the modeling of the roll-motion of a hull section, the propagation of fifth-order gravity waves and the piston wave-maker are outlined.

\subsection{Roll-motion of Hull-section}

For the roll-motion of a 2-D hull-section (see Fig. 1), $\mathbb{F}(t)$ and $\mathbb{H}(t)$ represent the instantaneous positions of the free-surface and hull surface respectively; $\boldsymbol{\Sigma}$ represents the far-field boundary used to truncate the infinite domain into a finite one and placed far enough from the hull to avoid reflection of the radiated waves. $\mathbf{n}$ (not shown in the Fig. 1) is the unit normal to a surface, positive inward. 


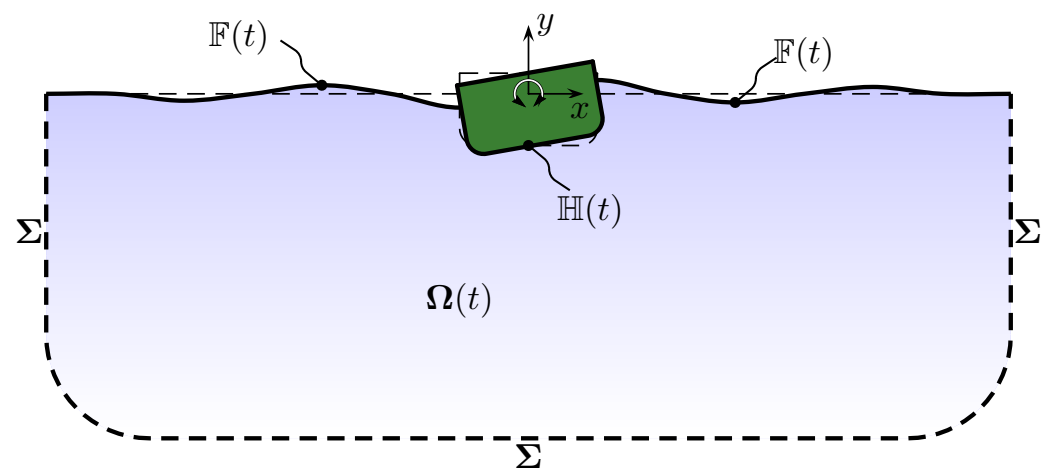

Figure 1: Hull-section roll-motion : Fluid domain and corresponding boundaries

\subsubsection{Boundary Conditions on Free-surface, $\mathbb{F}(t)$}

Fully nonlinear boundary conditions are imposed on the free-surface $\mathbb{F}(t)$. The dynamic boundary condition in the Lagrangian form is

$$
\frac{D \varphi}{D t}=\frac{1}{2}|\nabla \varphi|^{2}-g \eta-P_{\mathbb{F}} ; \quad \mathbf{x} \in \mathbb{F}(t)
$$

satisfied on the exact free-surface. In $(2), \frac{D}{D t} \equiv \frac{\partial}{\partial t}+\nabla \varphi \cdot \nabla$ denotes the material derivative, $\eta \equiv \eta(x, t)$ is the instantaneous free-surface and $P_{\mathbb{F}}$ is the pressure on the free-surface assumed to be zero.

An equally important free-surface boundary condition is the kinematic boundary condition also represented in the Lagrangian form as

$$
\frac{D \mathbf{x}}{D t}=\nabla \varphi ; \quad \mathbf{x} \in \mathbb{F}(t)
$$

\subsubsection{Boundary Condition on Hull, $\mathbb{H}(t)$}

On the hull surface $\mathbb{H}(t)$,

$$
\nabla \varphi \cdot \mathbf{n}=\mathbf{V}(\mathbf{x}, t) \cdot \mathbf{n} ; \quad \mathbf{x} \in \mathbb{H}(t)
$$

$\mathbf{V}(\mathbf{x}, t)$ is the prescribed motion of the hull. In the case of roll, the hull is subject to a forced sinusoidal angular motion of the form

$$
\alpha(t)=\alpha_{0} \sin (\omega t)
$$

where $\alpha_{0}$ is the amplitude of roll motion and $\omega$ is the corresponding circular frequency. In terms of Cartesian components, the prescribed roll motion is 


$$
\mathbf{V}(\mathbf{x}, t)=(-y \dot{\alpha}, x \dot{\alpha})
$$

\subsubsection{Boundary Condition on Far Field Boundary, $\Sigma$}

The far-field boundary $\boldsymbol{\Sigma}$ is assumed to be a no-flux surface and the corresponding boundary condition is

$$
\nabla \varphi \cdot \mathbf{n}=0 ; \quad \mathbf{x} \in \mathbf{\Sigma}
$$

Special attention is paid to place the boundary far away from the body to avoid reflection of the waves generated by the hull motion. The simulation is terminated before the waves reach the far-field boundary.

\subsubsection{Initial Conditions}

At time $t=0$, the relevant initial conditions are

$$
\left.\begin{array}{l}
\varphi(\mathbf{x}, 0)= \\
\eta(x, 0)= \\
=
\end{array}\right\} \mathbf{x} \in \mathbb{F}(t)
$$

\section{$2.2 \quad$ Fifth-order Gravity Waves}

The fluid domain $\boldsymbol{\Omega}(t)$ and its corresponding boundaries are as shown in Fig. 2 . $\mathbb{F}(t)$ represents the instantaneous position of the free-surface, $\mathbb{U}(t)$ and $\mathbb{D}(t)$ are the upstream and downstream periodic boundaries of the domain, and $\mathbb{B}(t)$ represents an impervious bottom surface.

The boundary condition on the free-surface $\mathbb{F}(t)$ are identical to that applied in the case of the roll-motion of a hull-section.

\subsubsection{Boundary Condition on $\mathbb{U}(t), \mathbb{D}(t)$}

$\mathbb{U}(t)$ and $\mathbb{D}(t)$ represent the periodic inflow and outflow wave boundaries respectively. In the modeling of the propagation of the Stokes waves, kinematic boundary conditions of the form

$$
\begin{array}{llr}
\mathbb{U}(t): & \nabla \varphi \cdot \mathbf{n}= & u_{w} \\
\mathbb{D}(t): & \nabla \varphi \cdot \mathbf{n}= & -u_{w}
\end{array}
$$

are imposed on these surfaces. Here $\mathbf{n}$ is the unit surface normal positive inward. $u_{w}$ is the horizontal velocity component corresponding to a fifth-order Stokes wave and has the form 


$$
u_{w}=u_{w}^{*} \sum_{n=1}^{5} \epsilon^{n} n a_{n} \cosh (n k s) \cos (n \theta)
$$

where $s=d+y$ and $\theta=2 \pi\left(\frac{x}{L}-\frac{t}{T}\right)$ with $T$ being the wave period and $L$ the wavelength; $\epsilon=\frac{k H}{2}$ is the dimensionless expansion parameter with $H$ being the wave height and $k=\frac{2 \pi}{L}$ the wavenumber; $a_{n}$ are coefficients that are functions of $\epsilon$ and $\frac{d}{L} ; u_{w}^{*}$ is the dimensional scaling velocity. This form is consistent with the fifth-order gravity wave theory of Fenton [1985]. For brevity only the functional form is presented here and a more detailed explanation of the terms in (10) can be found in Fenton [1985].

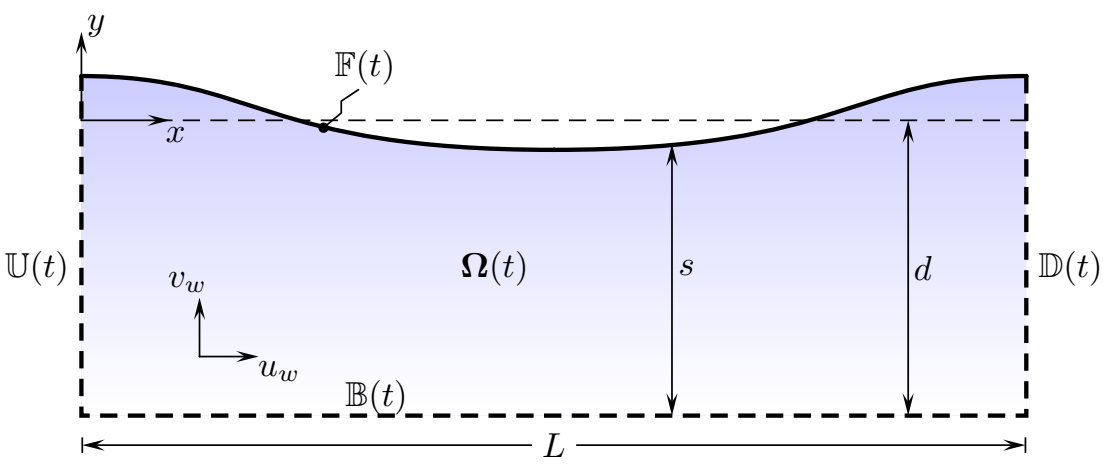

Figure 2: Fifth-order Stokes Wave : Fluid domain and corresponding boundaries, $d$ : water depth, $L$ : wavelength, $y$ : wave elevation, $s=d+y$

\subsubsection{Boundary Condition on $\mathbb{B}(t)$}

The bottom surface is assumed to be immovable and impervious and the corresponding boundary condition is

$$
\nabla \varphi \cdot \mathbf{n}=0 ; \quad \mathbf{x} \in \mathbb{B}(t)
$$

\subsubsection{Initial Conditions}

At time $t=0$, i.e., at the beginning of the simulation, the following conditions are applied on the free-surface

$$
\left.\begin{array}{rl}
\varphi(\mathbf{x}, 0) & =\varphi_{w}(\mathbf{x}, 0) \\
\eta(x, 0) & =\eta_{w}(x, 0)
\end{array}\right\} \mathbf{x} \in \mathbb{F}(t)
$$


where $\varphi_{w}$ and $\eta_{w}$ are the velocity potential and wave elevation corresponding to a fifth-order Stokes wave. The functional forms of $\varphi_{w}$ and $\eta_{w}$ are described in detail in Fenton [1985].

\subsection{Piston Wavemaker}

The fluid domain $\boldsymbol{\Omega}(t)$ corresponding to a numerical wave tank with a piston wavemaker is as shown in Fig. 3. $\mathbb{W}(t)$ represents the instantaneous position of the wavemaker and has a sinusoidal horizontal translation of the form

$$
x_{p}(t)=-\frac{A}{2} \cos (\omega t)
$$

where $A$ is the amplitude or stroke of the piston wavemaker and $\omega$ is the circular frequency of the sinusoidal motion. $\mathbb{F}(t)$ is the instantaneous free-surface; $\mathbb{B}(t)$ is the impervious bottom surface; $\mathbb{D}$ is the downstream wall of the wave tank.

The boundary condition on the free-surface $\mathbb{F}(t)$ are identical to that applied in the case of the previous two cases and is not repeated here for brevity.

\subsubsection{Boundary Condition on Wave-maker, $\mathbb{W}(t)$}

Along the piston surface, a kinematic boundary condition of the form

$$
\nabla \varphi \cdot \mathbf{n}=u_{p}(t) ; \quad \mathbf{x} \in \mathbb{W}(t)
$$

is applied, where $\mathbf{n}$ is the unit surface normal pointing into the fluid domain. From the sinusoidal motion of the piston as specified by (14), we have

$$
u_{p}(t)=\dot{x}_{p}(t)=\frac{A}{2} \omega \sin (\omega t)
$$

\subsubsection{Boundary Condition on $\mathbb{B}(t), \mathbb{D}$}

Both the bottom and downstream surfaces are assumed to be no-flux surfaces and the following kinematic boundary condition is applied

$$
\nabla \varphi \cdot \mathbf{n}=0 ; \quad \mathbf{x} \in \mathbb{B}(t), \mathbb{D}
$$

\subsubsection{Initial Conditions}

At time $t=0$, the relevant initial conditions are

$$
\left.\begin{array}{l}
\varphi(\mathbf{x}, 0)=0 \\
\eta(x, 0)=0
\end{array}\right\} \mathbf{x} \in \mathbb{F}(t)
$$




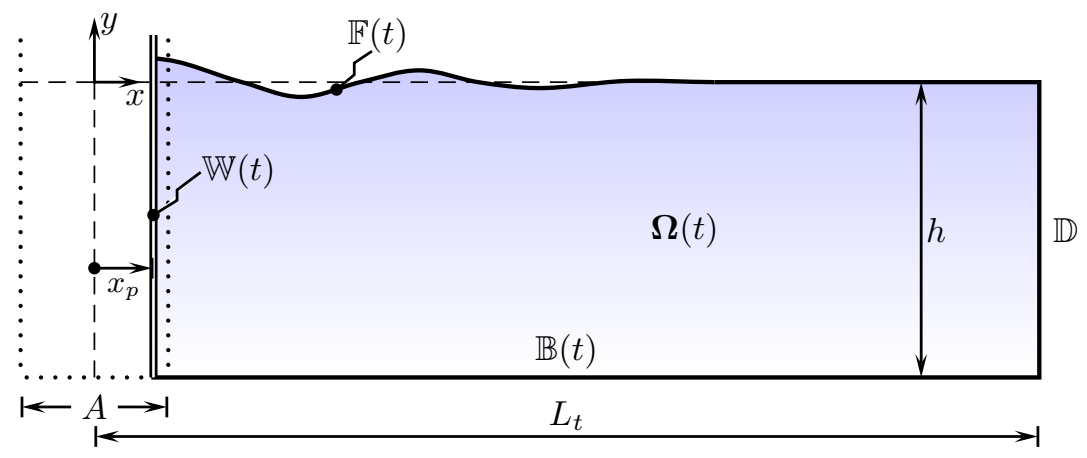

Figure 3: Piston Wavemaker : Fluid domain and corresponding boundaries, $h$ : wave tank depth, $A$ : Piston stroke, $L_{t}$ : Mean length of wave-tank

It can be observed from the sinusoidal motion, as specified by (13), that the wavemaker is at a position of extreme displacement of $x_{p}(0)=-\frac{A}{2}$ at time $t=0$. This arrangement is introduced to prevent an impulsive start to the simulation with non-zero velocities at the wavemaker.

\subsection{Boundary Integral Equation}

The BVP for the velocity potential is converted into a Boundary Integral Equation (BIE) by introducing a Green's function $G(\mathbf{p}, \mathbf{q})=-\frac{1}{2 \pi} \ln r_{\mathbf{p q}}$ (satisfies the Laplace equation), where $r_{\mathbf{p q}}=|\mathbf{p}-\mathbf{q}|, \mathbf{p} \equiv \mathbf{p}(\mathbf{x})$ is the field point and $\mathbf{q} \equiv \mathbf{q}(\mathbf{x})$ is the source point. The BIE obtained by applying Green's third identity to $\varphi(\mathbf{x}, t)$ and $G(\mathbf{p}, \mathbf{q})$ is

$$
\alpha(\mathbf{p}) \varphi(\mathbf{p})+\int_{\boldsymbol{\Gamma}} \varphi(\mathbf{q}) \frac{\partial G(\mathbf{p}, \mathbf{q})}{\partial n(\mathbf{q})} d \boldsymbol{\Gamma}_{\mathbf{q}}=\int_{\boldsymbol{\Gamma}} G(\mathbf{p}, \mathbf{q}) \frac{\partial \varphi(\mathbf{q})}{\partial n(\mathbf{q})} d \boldsymbol{\Gamma}_{\mathbf{q}}
$$

where $\mathbf{p} \in \boldsymbol{\Gamma}$ and $\boldsymbol{\Gamma}$ represents the boundary of the fluid domain, $\boldsymbol{\Gamma} \equiv \mathbb{F} \cup \mathbb{H} \cup \boldsymbol{\Sigma}$ or $\boldsymbol{\Gamma} \equiv \mathbb{F} \cup \mathbb{U} \cup \mathbb{B} \cup \mathbb{D}$ or $\boldsymbol{\Gamma} \equiv \mathbb{F} \cup \mathbb{W} \cup \mathbb{B} \cup \mathbb{D}$ depending on the type of simulation; $\alpha(\mathbf{p})$ is the internal angle formed at the boundaries (a coefficient introduced as a result of the exclusion of the singular nature of the Green's function). 


\section{Numerical Formulation}

Two important aspects that dominate the numerical formulation of the problem are (1) the solution of the boundary integral equation (18) and, (2) the time integration of the free-surface boundary conditions (2) and (3).

In the BEM model presented here, a Mixed Eulerian Lagrangian (MEL) scheme of Longuet-Higgins and Cokelet [1976] is used to solve the boundary value problem with the fully nonlinear free-surface boundary conditions. The MEL scheme comprises of two steps :(1) solve a well-defined boundary value problem with Neumann or Dirichlet type boundary conditions using BEM; (2) advance the free-surface in time using the time dependent free-surface boundary conditions. These two steps are repeated at each step of a higher-order time marching scheme.

\subsection{Solution of BIE}

Linear iso-parametric elements form the basis for the numerical solution of the BIE (18). The variation of $\varphi$ and $q \equiv \frac{\partial \varphi}{\partial n}$ within each element, and the geometry of the element are defined as, following the notation given in Wrobel [2002] and Brebbia [1978],

$$
\left.\begin{array}{l}
\varphi(\xi)=N_{1}(\xi) \varphi_{1}+N_{2}(\xi) \varphi_{2} \\
q(\xi)=N_{1}(\xi) q_{1}+N_{2}(\xi) q_{2}
\end{array}\right\}
$$

and,

$$
\left.\begin{array}{l}
x(\xi)=N_{1}(\xi) x_{1}+N_{2}(\xi) x_{2} \\
y(\xi)=N_{1}(\xi) y_{1}+N_{2}(\xi) y_{2}
\end{array}\right\}
$$

where $\left(\varphi_{1}, q_{1}\right)$ and $\left(\varphi_{2}, q_{2}\right)$ are the values of the potential and the flux at the two nodal points of the element. To ensure the compatibility of the potential and flux between the elements, the two nodal points are chosen to be the end-points of the element specified by the coordinate pairs $\left(x_{1}, y_{1}\right)$ and $\left(x_{2}, y_{2}\right)$ respectively. With the nodal points at the end-points of the element, the interpolation or shape functions $N_{1}(\xi)$ and $N_{2}(\xi)$ can be written in the form

$$
N_{1}(\xi)=\frac{1}{2}(1-\xi) ; N_{2}(\xi)=\frac{1}{2}(1+\xi)
$$

where $\xi$ is the intrinsic element coordinate, $-1 \leq \xi \leq+1$.

Based on the approximations (19) and (20), the discretized form of (18) can be written as, applied at a node $i$

$$
\alpha_{i} \varphi_{i}+\sum_{j=1}^{N_{e}} \int_{S_{j}} q^{*}\left(N_{1} \varphi_{1}+N_{2} \varphi_{2}\right) d S_{j}=\sum_{j=1}^{N_{e}} \int_{S_{j}} \varphi^{*}\left(N_{1} q_{1}+N_{2} q_{2}\right) d S_{j}
$$


where $q^{*} \equiv \frac{\partial G(p, q)}{\partial n(q)}$ and $\varphi^{*} \equiv G(p, q) ; N_{e}$ is the total number of boundary elements. Following the notation given in Wrobel [2002], the discretized form of the BIE in eq. (22) can be written in a matrix form

$$
\mathrm{H} \varphi=\mathrm{GQ}
$$

where $\mathbf{H}$ and $\mathbf{G}$ are the influence coefficient matrices; $\boldsymbol{\varphi}$ and $\mathbf{Q}$ are the vectors containing the nodal values of the potential and its normal derivatives. The elements of the influence coefficient matrices have the form

$$
\left.\begin{array}{rl}
G_{i j} & =g_{i, j-1}^{2}+g_{i, j}^{1} \\
\hat{H}_{i j} & =h_{i, j-1}^{2}+h_{i, j}^{1}
\end{array}\right\}
$$

where,

$$
g_{i j}^{k}=\int_{S_{j}} \varphi^{*} N_{k} d S_{j} \quad ; \quad h_{i j}^{k}=\int_{S_{j}} q^{*} N_{k} d S_{j}
$$

The integrals in eq. (25) are evaluated analytically to evaluate the influence coefficient matrices. An important aspect in the use of higher-order boundary elements is the treatment of discontinuity in the flux $q$ at corners (geometric discontinuities) of the domain. In the current BEM scheme, a "double node" approach is used to increase the number of degrees of freedom at a corner to three. This approach assumes that the potential is continuous but allows for the specification of discontinuous fluxes before and after the node. However, out of the three degrees of freedom, two are eliminated based on the boundary conditions unique to the simulation under consideration. The linear system of equations in (23) is re-ordered based on either Neumann or Dirichlet type boundary conditions specified on the domain boundaries and can be written in the form

$$
\mathbf{A X}=\mathbf{F}
$$

where $\mathbf{X}$ is the vector of unknowns and $\mathbf{F}$ is the so called "load" vector (Wrobel [2002]). A direct LU-solver (Press et al. [1992]) is used to solve the re-ordered system of equations.

\subsection{Time Integration}

A strictly Lagrangian approach is used for the time-integration of the free-surface kinematic and dynamic boundary conditions. The free-surface boundary conditions (2) and (3), are of the general form

$$
\frac{D Y}{D t}=f(t, Y)
$$


where, $Y$ can be either $\mathbf{x}$ or $\varphi$. A fourth-order Runge-Kutta (RK4) scheme, as shown below, is used to integrate (27) in time.

$$
\left\{\begin{aligned}
k_{1} & =\Delta t f\left(t_{i}, Y_{i}\right) \\
k_{2} & =\Delta t f\left(t_{i}+0.5 \Delta t, Y_{i}+0.5 k_{1}\right) \\
k_{3} & =\Delta t f\left(t_{i}+0.5 \Delta t, Y_{i}+0.5 k_{2}\right) \\
k_{4} & =\Delta t f\left(t_{i+1}, Y_{i}+k_{3}\right) \\
Y_{i+1} & =Y_{i}+\frac{1}{6}\left(k_{1}+2 k_{2}+2 k_{3}+k_{4}\right)
\end{aligned}\right.
$$

where $\Delta t$ is the discrete time-step. It may be noted that a BEM-step is solved at each step of the RK4 time integration scheme.

\subsection{Free-surface Corners}

For hull roll-motion and wavemaker simulations, the intersection between the freesurface and the moving structure has to be treated carefully to avoid numerical instabilities. In addition to the "double-node" approach, velocity compatibility conditions (see Grilli and Svendsen [1990]) are imposed at the intersection. These conditions have the form

$$
\begin{aligned}
\varphi_{s}^{\mathbb{S}} & =\frac{\varphi_{n}^{\mathbb{S}} \cos \left(\beta^{\mathbb{F}}-\beta^{\mathbb{S}}\right)-\varphi_{n}^{\mathbb{F}}}{\sin \left(\beta^{\mathbb{F}}-\beta^{\mathbb{S}}\right)} \\
\varphi_{s}^{\mathbb{F}} & =\frac{\varphi_{n}^{\mathbb{S}}-\varphi_{n}^{\mathbb{F}} \cos \left(\beta^{\mathbb{F}}-\beta^{\mathbb{S}}\right)}{\sin \left(\beta^{\mathbb{F}}-\beta^{\mathbb{S}}\right)}
\end{aligned}
$$

where $\varphi_{s}^{()}$and $\varphi_{n}^{()}$are the tangential and normal velocity components on the surface; $\beta^{()}$is a measure of the slope of the surface (see Fig. 4). The superscripts ()$^{\mathbb{F}}$ and ()$^{\mathbb{S}}$ represent the free-surface $\mathbb{F}(t)$ and the moving structure $\mathbb{H}(t)$ or $\mathbb{W}(t)$ respectively.

\section{Results}

The BEM scheme is applied to the following cases : (1) the propagation of fifthorder gravity waves, (2) transient waves generated by a piston wavemaker and (3) 2-D simulation of hull-section in roll-motion. The results and details of these simulations are described below.

\subsection{Comparison with Analytic Solution : Fifth-order Gravity Waves}

A wave with wave length $L=65 \mathrm{~m}$, wave height $H=5.0 \mathrm{~m}$, wave period $T=6.38 \mathrm{~s}$ and water depth $d=20 \mathrm{~m}\left(\frac{d}{g T^{2}}=0.05, \frac{H}{g T^{2}}=0.0125, \frac{H}{L}=0.0769\right)$ is chosen 


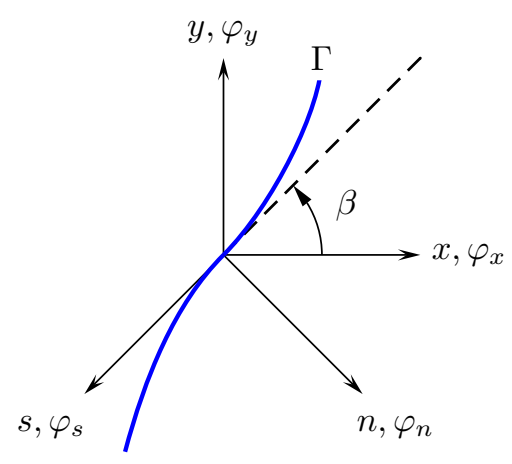

Figure 4: Orientation of orthogonal coordinate systems and velocity components on a boundary element surface; $(x, y)$ Cartesian coordinate system, $(s, n)$ tangential-normal coordinate system; $\Gamma$ : BEM surface

to simulate the propagation of periodic gravity waves. At the beginning of the simulation, the characteristics of the wave as obtained from analytical expressions of Fenton [1985] are specified as initial conditions on the free-surface. The BEM scheme is then allowed to propagate the wave based on the boundary conditions specified on the upstream and downstream surfaces, $\mathbb{U}(t)$ and $\mathbb{D}(t)$ respectively.

A comparison between the analytical $\left(\eta_{w}\right.$, Fenton [1985]) and numerical $\left(\eta_{n}\right)$ wave profiles is shown in Fig. 5. The simulation is carried out for $N_{\mathbb{F}}=240$ and $N_{\mathbb{T}}=500$ linear BEM panels with a time step $\Delta t=0.025 T$. Here, $N_{\mathbb{F}}$ is the total number of panels on the free-surface $\mathbb{F}$, and $N_{\mathbb{T}}$ is the total number of panels over the entire BEM domain $\mathbb{T} \equiv \mathbb{F} \cup \mathbb{D} \cup \mathbb{B} \cup \mathbb{U}$. The scheme is allowed to propagate the waves for a total simulation time of $5.0 T$, and Fig. 5 compares the analytical and numerical wave profiles between $t=4.0 T$ and $t=4.8 T$ at steps of $0.2 T$. It can be observed that the BEM scheme is able to preserve the form and characteristics of the initial wave profile over long periods of time.

The spatial and temporal convergence characteristics of the scheme are studied by comparing the analytical and numerical drifts of a Lagrangian fluid particle $\mathbf{P}[x(t), y(t)]$, placed at the intersection of the free-surface $\mathbb{F}(t)$ and the upstream boundary $\mathbb{U}(t)$. To determine the analytical drift (particle trajectory), the system of equations

$$
\frac{D x}{D t}=u_{w}(x, y, t) ; \frac{D y}{D t}=v_{w}(x, y, t)
$$

is numerically integrated using a fourth-order Runge-Kutte scheme. Here, $u_{w}$ and $v_{w}$ respectively are the horizontal and vertical components of the wave velocity (based on analytical expressions, Fenton [1985]). 


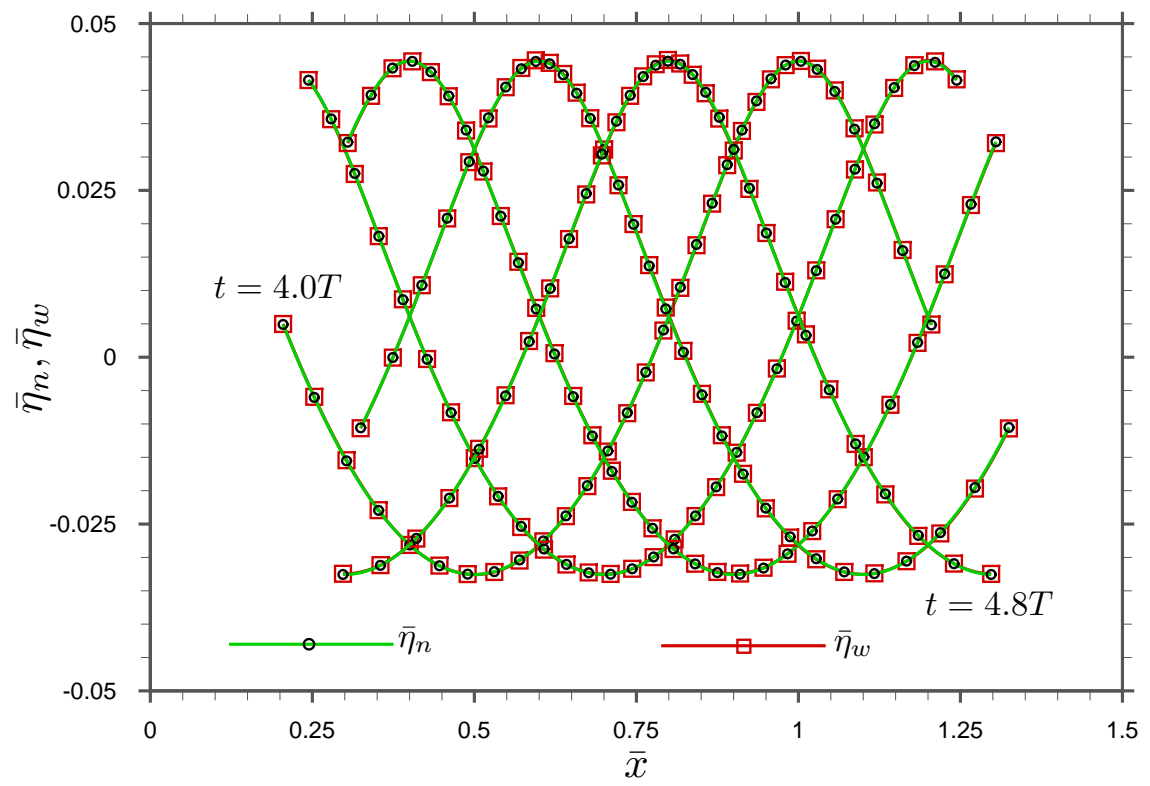

Figure 5: Comparison of numerical and analytical wave elevations between $t=4.0 T$ and $t=4.8 T$ at steps of $0.2 T ; \bar{x}=x / L, \bar{\eta}_{w}=\eta_{w} / L, \bar{\eta}_{n}=\eta_{n} / L: \eta_{n}:$ numerical wave elevation, $\eta_{w}$ : analytical wave elevation; BEM characteristics : Time step $\Delta t / T=0.025$, Number of panels on the free-surface, $N_{\mathbb{F}}=240$

The trajectory and time-history of the fluid particle $\mathbf{P}$ and its convergence towards the analytical values are shown in Fig. 7 and Fig. 6 respectively. These figures summarize the results of simulations performed over a duration of $5.0 T$ for $N_{\mathbb{F}}=30,60,120,240\left(N_{\mathbb{T}}=75,150,300,500\right)$ and $\Delta t=0.0125 T, 0.025 T$. To quantify the convergence characteristics of the BEM scheme, the error between the analytical and numerical particle trajectories is compared at the end of the simulation, $t=5.0 T$, for different levels of discretization and time-steps. It is observed that (see Fig. 8) for $\Delta t=0.0125 T$ and $\Delta t=0.025 T,\left|\epsilon_{x}(t)\right| \approx \mathcal{O}\left(\Delta \bar{x}^{1.1}\right)$ and $\left|\epsilon_{y}(t)\right| \approx \mathcal{O}\left(\Delta \bar{x}^{1.25}\right)$. Here, $\epsilon_{x}(t)$ and $\epsilon_{y}(t)$ are the errors between the analytical and numerical $\bar{x}(t) \equiv x(t) / L$ and $\bar{y}(t) \equiv y(t) / L$ respectively, $\Delta \bar{x}=\frac{1}{N_{\mathbb{F}}}$ is a measure of the panel length.

It is important that the BEM scheme conserve mass and for free-surface simulations this property can be quantified by monitoring the area under the free-surface $\eta_{\text {area }}$ (ideally $\eta_{\text {area }}=0$ ). Fig. 9 illustrates the time-history of $\bar{\eta}_{\text {area }}=\eta_{\text {area }} / L^{2}$ for different levels of discretization. The maximum error in $\bar{\eta}_{\text {area }}$ is observed to be $3 \times 10^{-2 \%}$ and $6.5 \times 10^{-4 \%}$ for $N_{\mathbb{F}}=30$ and $N_{\mathbb{F}}=240$ respectively. 

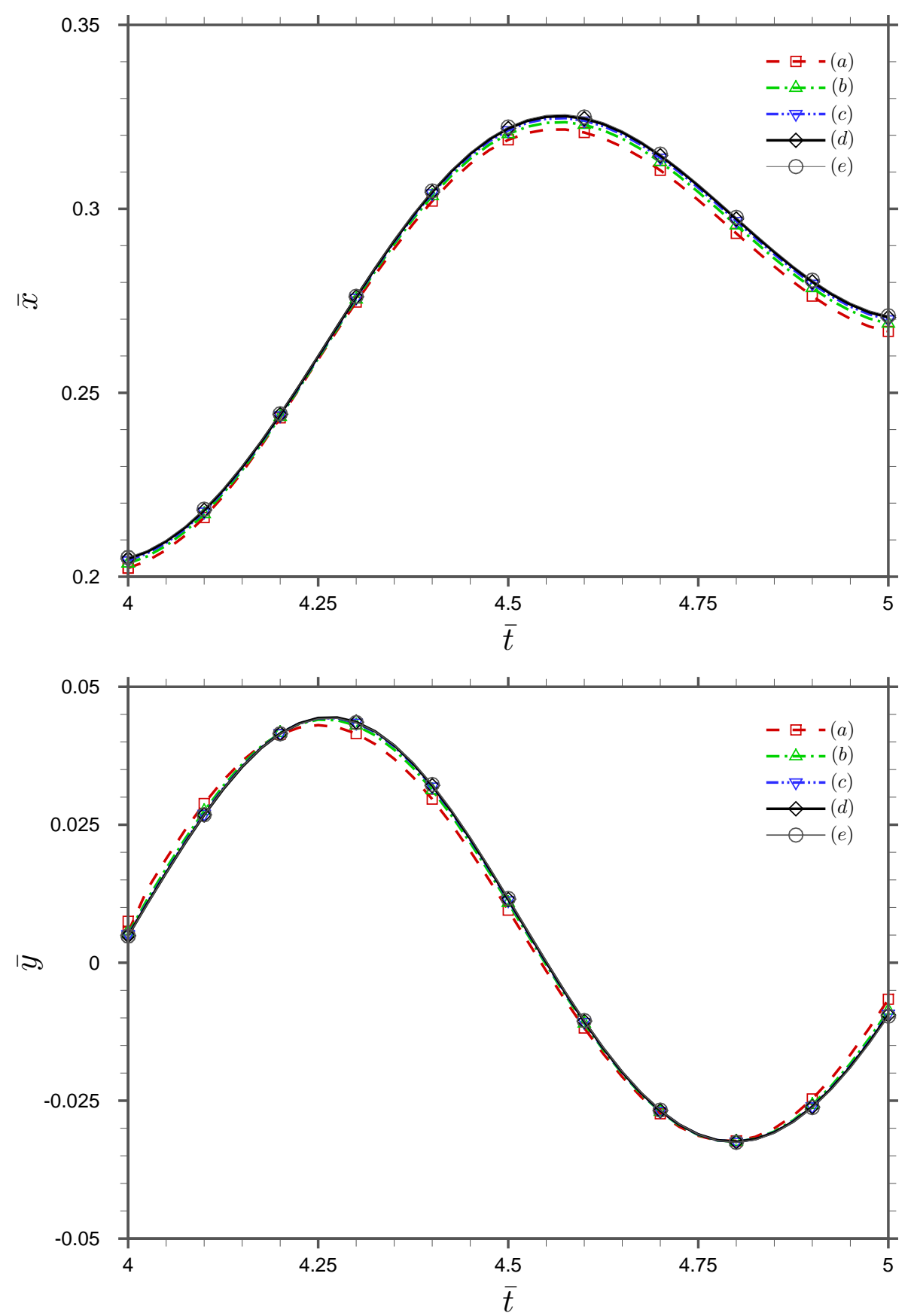

Figure 6: Time history : Convergence of the trajectory of a fluid particle $\mathbf{P}[x(t), y(t)]$ at the upstream boundary with increase in $N_{\mathbb{F}}$, number of panels on the free-surface: $(a) N_{\mathbb{F}}$ $=30,(b) N_{\mathbb{F}}=60,(c) N_{\mathbb{F}}=120,(d) N_{\mathbb{F}}=240,(e)$ Analytical trajectory; Time step $\Delta t / T$ $=0.025 ; \bar{x}=x / L, \bar{y}=y / L, \bar{t}=t / T$ 


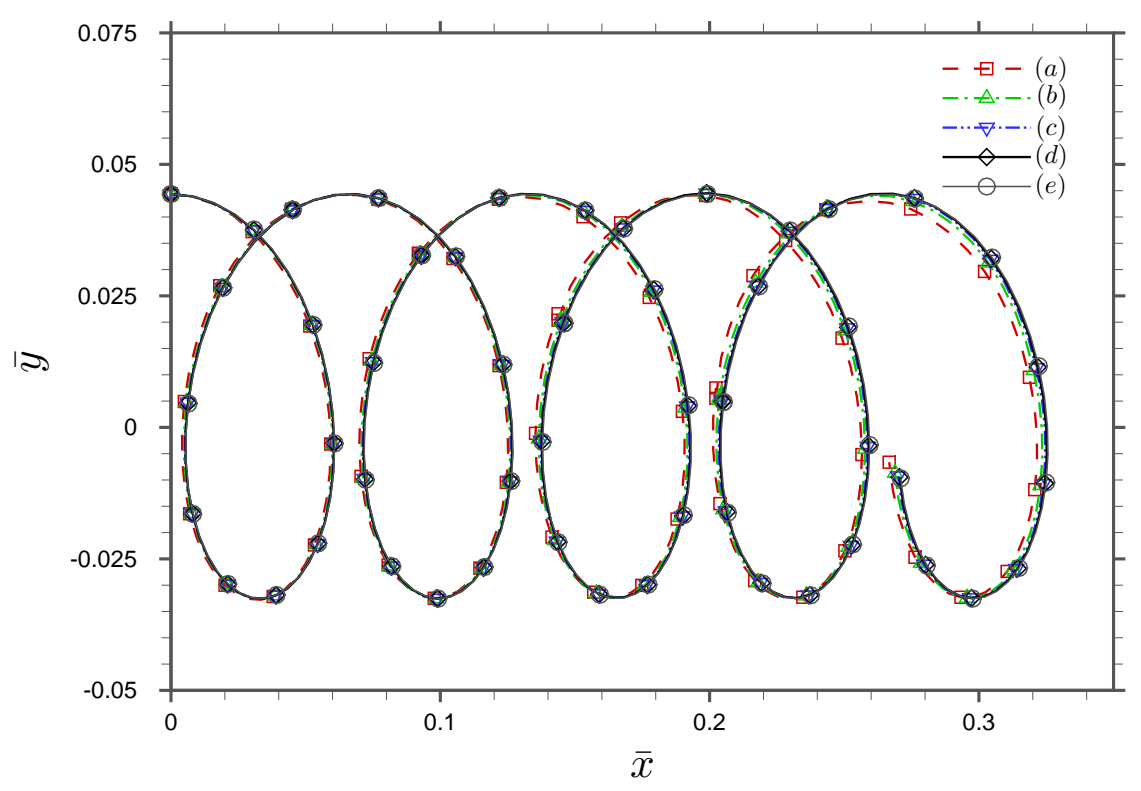

Figure 7: Convergence of the trajectory of a fluid particle $\mathbf{P}[x(t), y(t)]$ at the upstream boundary with increase in $N_{\mathbb{F}}$, Number of panels on the free-surface : (a) $N_{\mathbb{F}}=30,(b) N_{\mathbb{F}}$ $=60,(c) N_{\mathbb{F}}=120,(d) N_{\mathbb{F}}=240,(e)$ Analytical trajectory; Time step $\Delta t / T=0.025$; $\bar{x}=x / L, \bar{y}=y / L, \bar{t}=t / T$
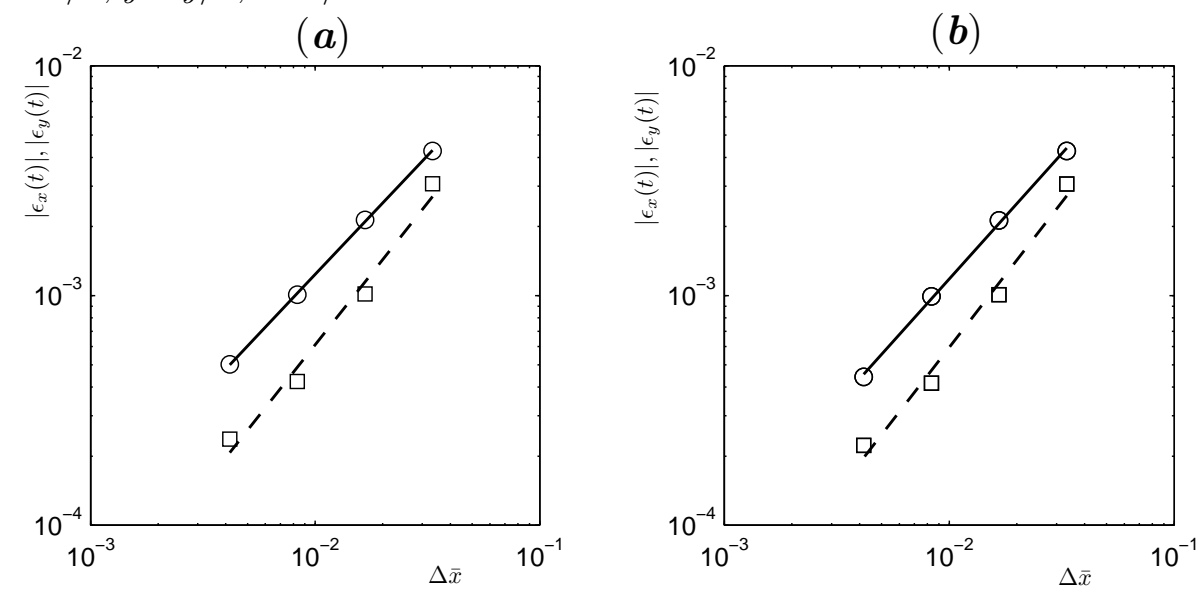

Figure 8: Convergence characteristics of the error between the analytical and numerical particle trajectories at time $t=5.0 T$ for $(\boldsymbol{a}) \Delta t=0.025 T$, and $(\boldsymbol{b}) \Delta t=0.0125 T ;(-\circ-)$ $\epsilon_{x}(t):$ error in $x(t),(-\square-) \epsilon_{y}(t):$ error in $y(t), \Delta \bar{x}=\frac{1}{N_{\mathbb{F}}}\left(N_{\mathbb{F}}=30,60,120,240\right)$ 


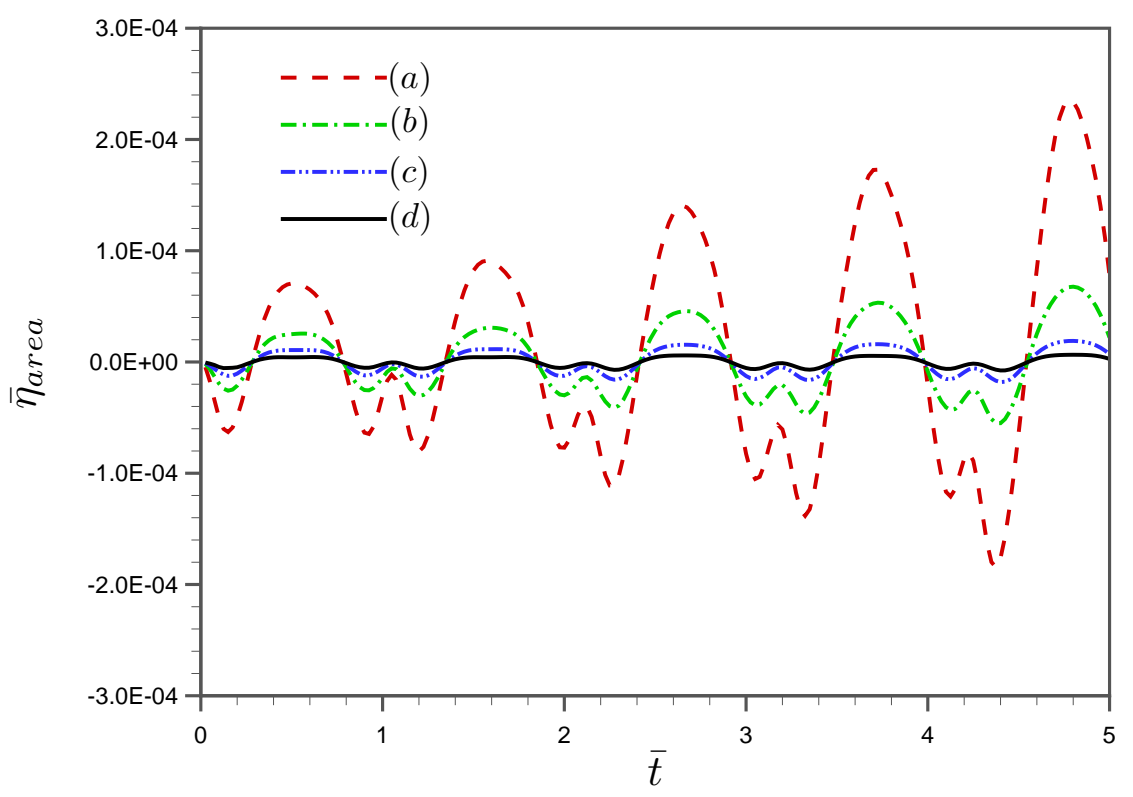

Figure 9: Variation of the area under the free-surface with time; Convergence with increase in $N_{\mathbb{F}}$, number of panels on the free-surface: $(a) N_{\mathbb{F}}=30,(b) N_{\mathbb{F}}=60,(c) N_{\mathbb{F}}=120,(d)$ $N_{\mathbb{F}}=240 ; \bar{\eta}_{\text {area }}=\eta_{\text {area }} / L^{2}:$ area under the free-surface; Time step $\Delta t / T=0.025$

\subsection{Comparison with other Numerical Method : Piston Wavemaker}

The next stage in the verification of the BEM scheme is the study of the transient waves generated by a piston wavemaker and forms an intermediate step in the application of the scheme to wave-body interactions. The numerical results from Lin [1984], based on the solution of the Cauchy's Integral Theorem, provide a basis for the verification of the transient waves generated by the wavemaker. For the simulation, a wave tank of depth $h=1.0$ is chosen with a mean length of $L_{t}=10 h$. The piston wavemaker placed at the left end of the tank has a stroke $A=0.1 h$ and oscillates with a period of $T=4.0(\omega=0.5 \pi)$. These quantities, in non-dimensional form, are consistent with the parameters specified in Lin [1984].

Fig. 10 compares the transient wave elevations between Lin [1984] and the BEM scheme at $t=2 T$ and $t=4 T$ (the intermediate steps are not presented for clarity). The simulations are performed with $N_{\mathbb{F}}=200$ and time steps $\Delta t=0.02 T$ and $\Delta t=0.01 T$. The overall comparison between the two methods is good and a detailed analysis of the wavemaker is not undertaken as its sole purpose is to verify the accuracy of the BEM scheme. 

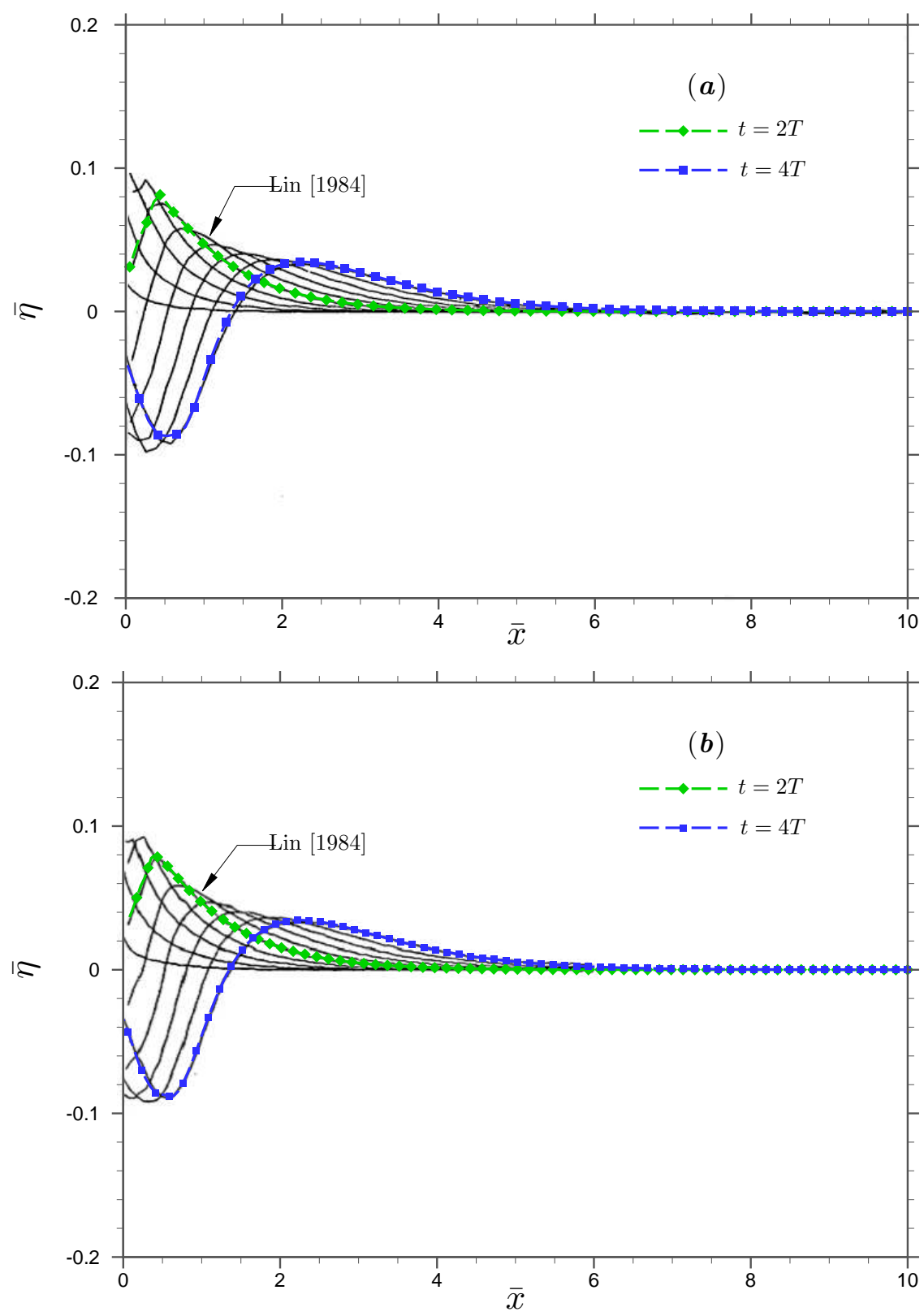

Figure 10: Piston wave-maker : Comparison of wave elevations between Lin [1984] and BEM scheme $(\boldsymbol{a}) \Delta t / T=0.02,(\boldsymbol{b}) \Delta t / T=0.01 ; \bar{A}=0.1, T=4.0, N_{\mathbb{F}}=200 ;$ $\bar{\eta}=\eta / h:$ wave elevation, $\bar{x}=x / h$ 


\subsection{Hull-section in Roll Motion}

The BEM scheme verified in the previous two sections is now applied to the rollmotion of a 2-D hull section. Two important parameters that govern the roll-motion of the hull-section are (1) the amplitude of roll-motion, $\alpha_{0}$ and (2) the Froude number, $\tilde{\omega}$, representing the non-dimensional frequency of roll-motion and

$$
\tilde{\omega}=\omega \sqrt{\frac{b}{g}}
$$

where $\omega(\mathrm{rad} . / \mathrm{s})$ is the frequency of roll-motion and $b=0.5 B$, with $B$ being the breadth of the hull-section.

The primary aim of the scheme is to ascertain the extent to which linear freesurface boundary conditions are applicable and the results presented here focus on this aspect. A representative $\tilde{w}=0.6$ is chosen and the effects of non-linearity are analyzed for different amplitudes of roll-motion. Fig. 11 and Fig. 12 illustrate the position of the hull and the free-surface elevation for a fully non-linear simulation with $\alpha_{0}=0.40 \mathrm{rad} .\left(\approx 23^{\circ}\right)$ over a duration of $8 T$, where $T=\frac{2 \pi}{\omega}$ is the period of oscillation. Fig. 12 also shows a few Lagrangian fluid particles that are tracked to model the free-surface. For both the port and starboard side free-surfaces, 400 BEM panels are used over a length of $\approx 100 B$. The panels are distributed to have a dense distribution close to the hull and a coarse one further away to increase the computational efficiency. A time-step of $\Delta t=0.01 T$ is used for the fourth-order Runge-Kutta scheme.

A comparison of the free-surface elevations obtained from the linear and nonlinear versions of the BEM scheme is shown in Fig. 13. For $\alpha_{0}=0.05 \mathrm{rad}(\approx$ $\left.3^{\circ}\right)$, there is no discernible difference between the linear and non-linear free-surface elevations. However, this is not true in the case of $\alpha_{0}=0.40 \mathrm{rad}\left(\approx 23^{\circ}\right)$, where the effect of the non-linear free-surface boundary conditions is clearly evident with the presence of secondary waves of smaller wavelength. A similar observation is made in Fig. 14( $\boldsymbol{a})$, where the effect of the non-linear free-surface boundary conditions is shown to increase with the amplitude of roll. An alternate way to represent the effects of non-linearity is to scale the wave elevations with the amplitude of roll $\alpha_{0}$, see Fig. 14(b). Ideally for a linear phenomenon, the scaled free-surface elevations should be identical. It can be observed that up to an amplitude of roll of $\alpha_{0}=0.1$ $\operatorname{rad}\left(\approx 6^{\circ}\right)$, the effects of non-linearity are small.

A convergence test is also carried out to ascertain the grid-independence of the non-linear secondary waves seen in Fig. 14 by varying the number of elements on the free-surface. For $N_{\mathbb{F}}=400$ and $N_{\mathbb{F}}=500$, the free-surface elevations are identical in the vicinity of the hull and the presence of the secondary waves is still observed (see Fig. 15). Here, $N_{\mathbb{F}}$ is the number of BEM panels on the port or starboard free-surfaces. 


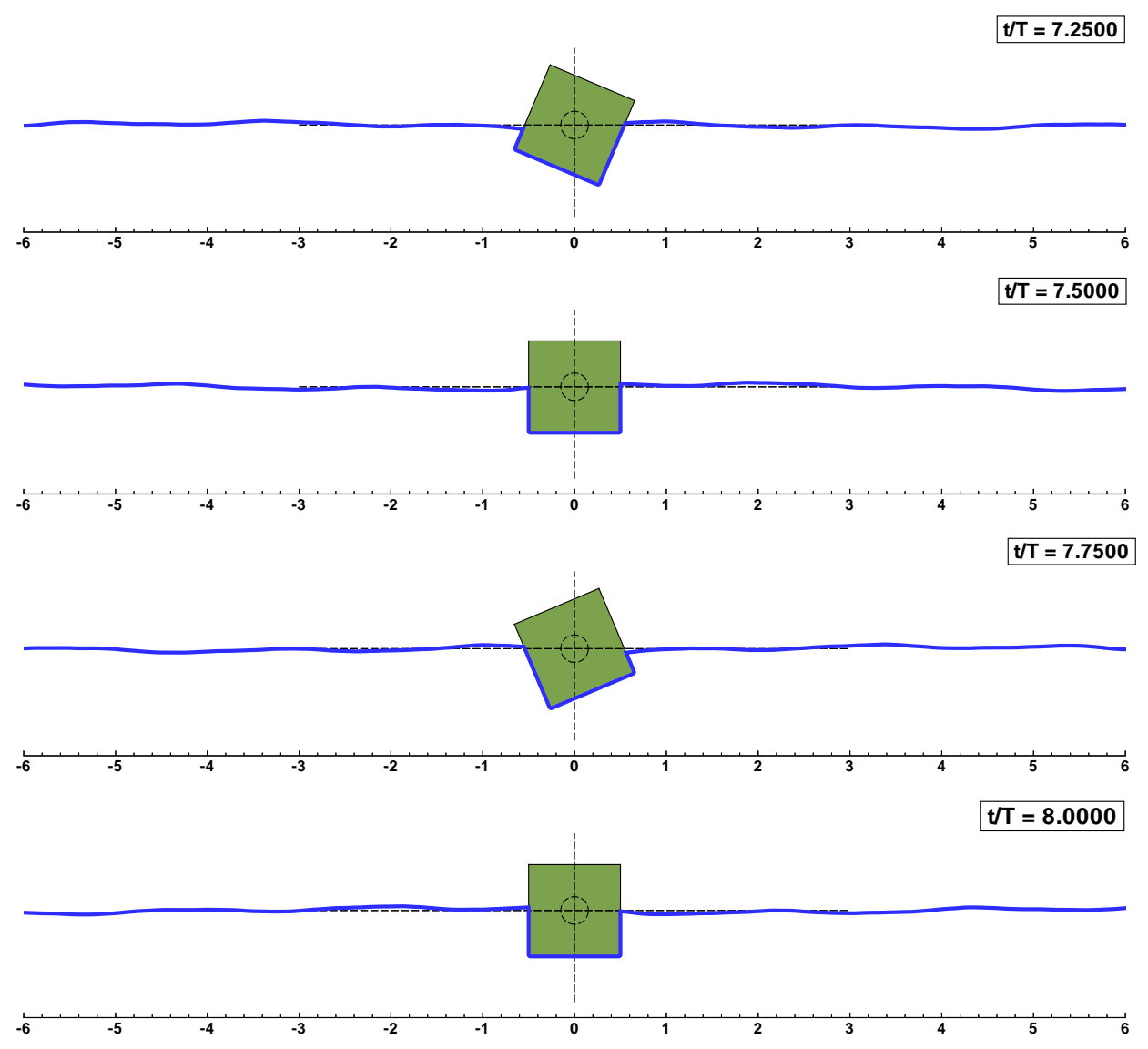

Figure 11: Hull-section and free-surface; $\tilde{\omega}=0.6, \alpha_{0}=0.4 \mathrm{rad} ;($ Note : the horizontal and vertical scales are identical) 

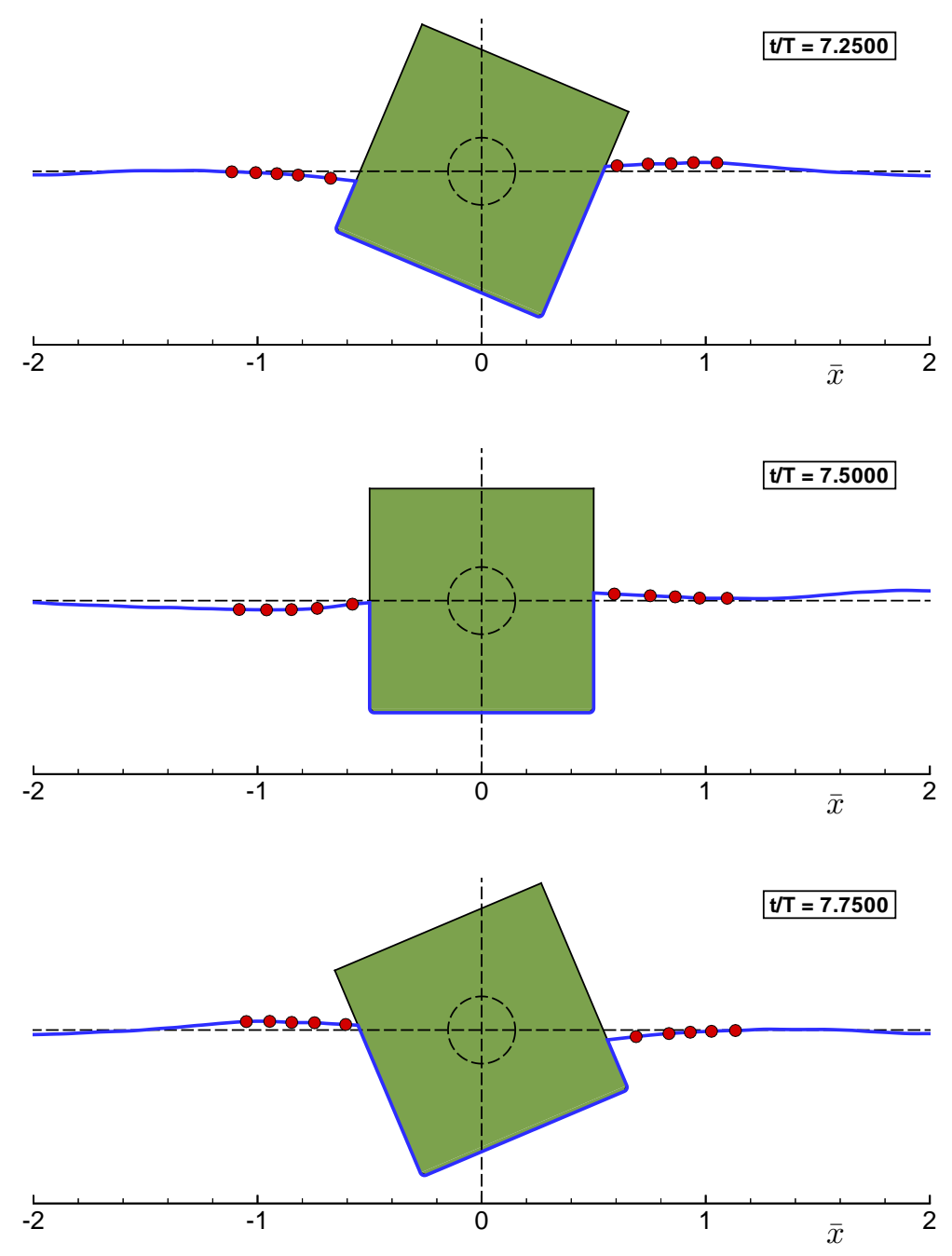

Figure 12: Hull-section and free-surface with actual Lagrangian fluid particles; $\tilde{\omega}=0.6$, $\alpha_{0}=0.4 \mathrm{rad} ; \bar{x}=x / B ;($ Note : the horizontal and vertical scales are identical) 

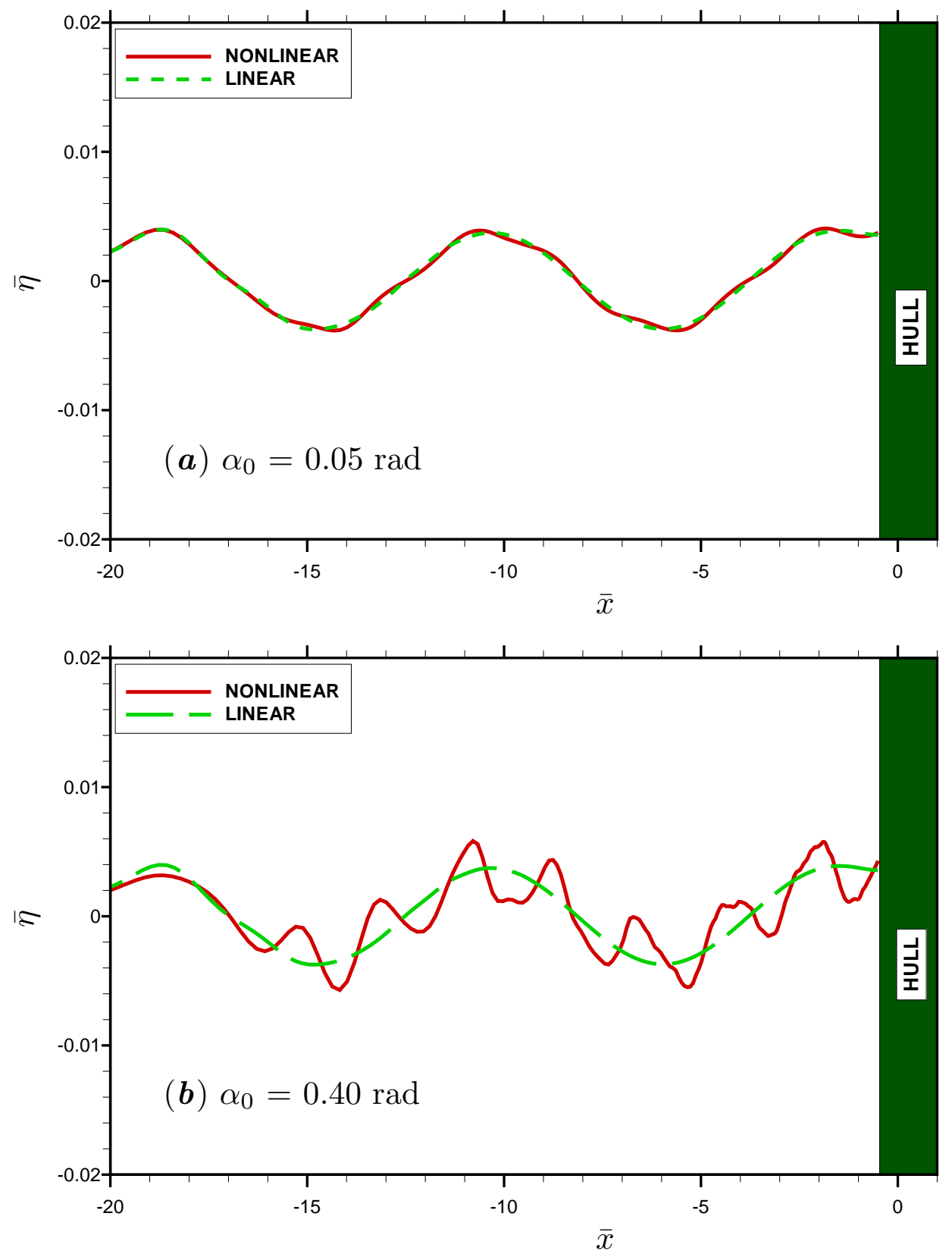

Figure 13: Comparison of linear and non-linear free-surface elevations; $(\boldsymbol{a}) \alpha_{0}=0.05 \mathrm{rad}$, (b) $\alpha_{0}=0.40 \mathrm{rad} ; \tilde{\omega}=0.6 ; N_{\mathbb{F}}=400$, Number of panels on the starboard free-surface; (Note: the horizontal and vertical scales are not identical) 

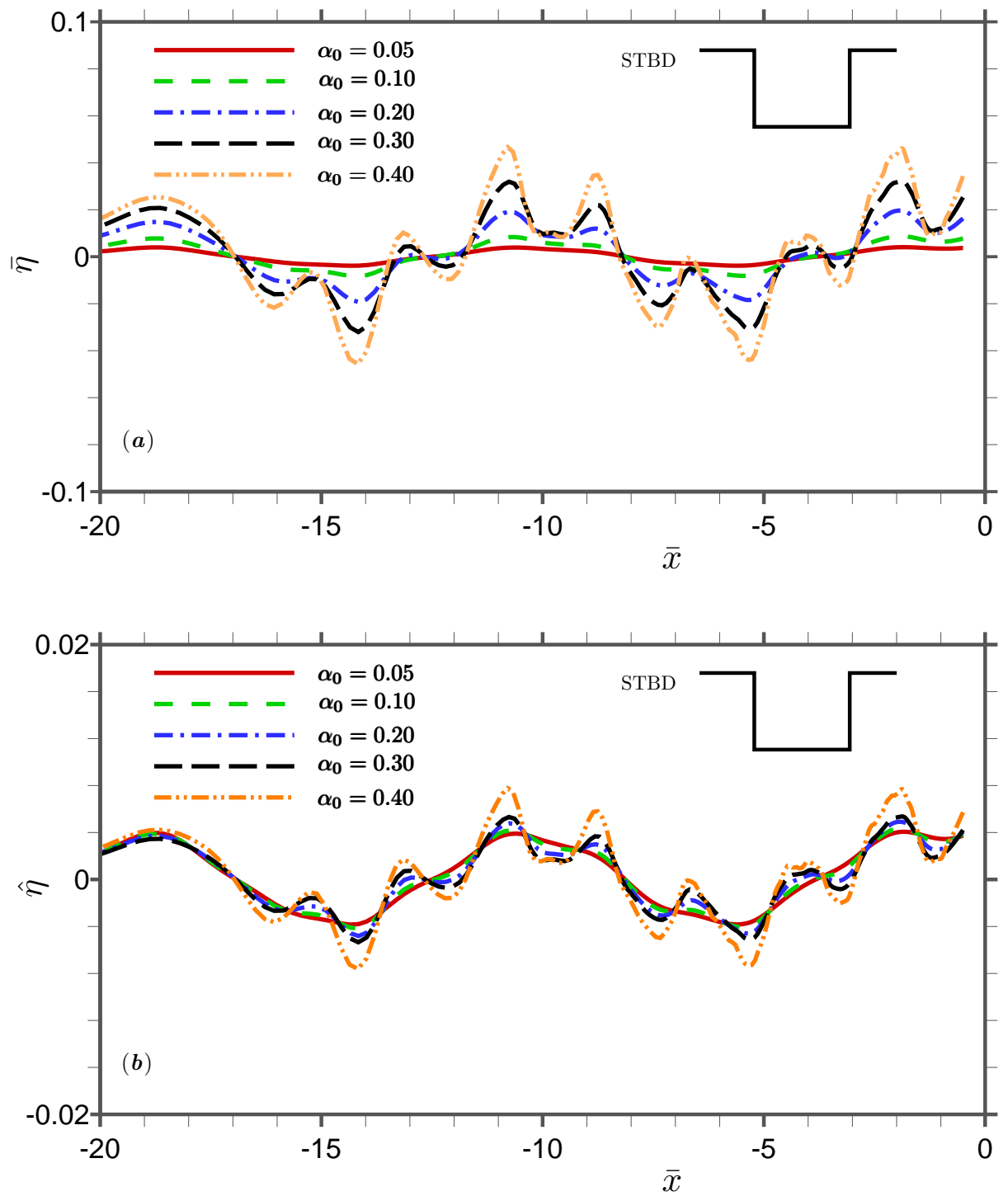

Figure 14: Scaled and un-scaled wave-elevations; $\tilde{\omega}=0.6 ; \bar{\eta}=\eta / B$ : free-surface elevation; $\hat{\eta}=\bar{\eta} \frac{0.05}{\alpha_{0}}$ scaled wave elevation; $\bar{x}=x / B ; N_{\mathbb{F}}=400$, Number of panels on the starboard free-surface; (Note : the horizontal and vertical scales are not identical) 


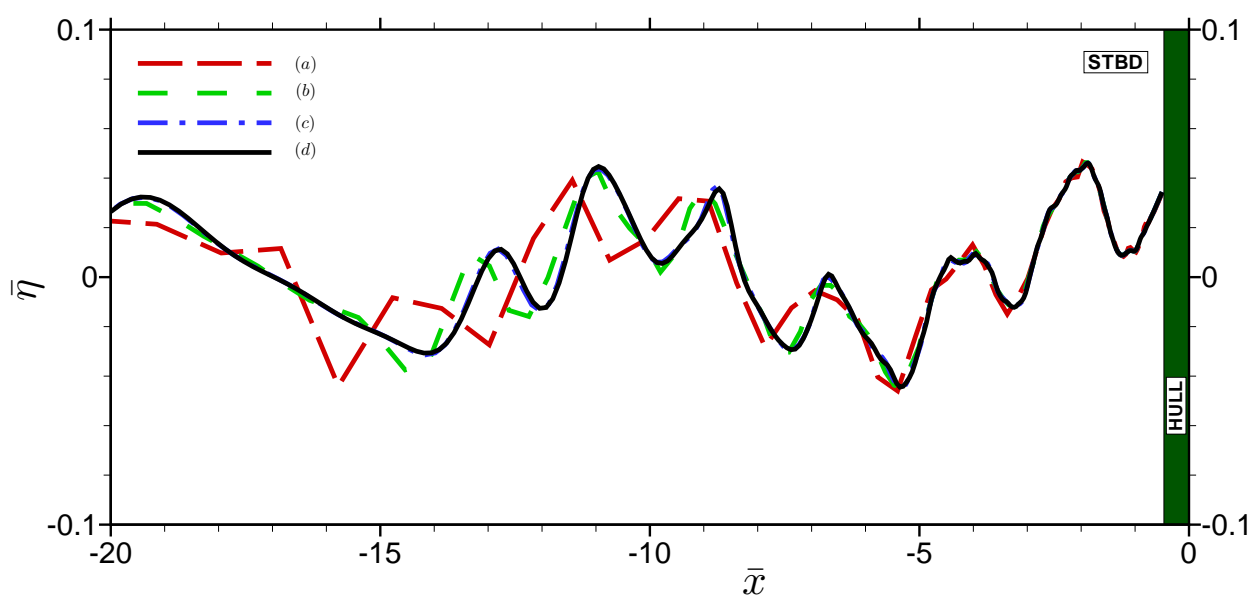

Figure 15: Convergence of free-surface elevations with increase in number of panels on the free-surface (a) $N_{\mathbb{F}}=100$ (b) $N_{\mathbb{F}}=200$ (c) $N_{\mathbb{F}}=400$ (d) $N_{\mathbb{F}}=500 ; N_{\mathbb{F}}$ is the number of panels on the starboard side free-surface (Note : the horizontal and vertical scales are not identical)

\section{Concluding Remarks}

A BEM scheme, coupled with a Mixed-Eulerian-Lagrangian approach, was developed with an intent to model the free-surface associated with the roll-motion of a 2-D hull section. Prior to its application to the roll-motion of the hull-section, the scheme was validated through an application to the propagation of fifth-order gravity waves (analytical method) and the transient waves generated by a piston wave-maker (alternate numerical method). The scheme was found to have a spatial rate of convergence of $\mathcal{O}(\Delta \bar{x})$ which is consistent with the use of linear BEM panels.

In the case of roll motion, the effects of nonlinearity was found to increase with the amplitude of roll motion. It was observed that, up to an amplitude of roll of $\alpha_{0}=0.1 \mathrm{rad}\left(\approx 6^{\circ}\right)$, the effects of non-linearity are small and the use of linear free-surface boundary conditions is justified. However, for amplitudes of roll $\alpha_{0}>0.1 \mathrm{rad}$, it is important to follow a non-linear approach to the modeling of the free-surface.

The current scheme can be extended further to analyze a wide-range of application of 2-D and 3-D wave-body interactions. The applications include the development of a numerical wave tank to analyze the response of a hull in an ambient wave environment. 


\section{Acknowledgement}

This research has been supported by the Offshore Technology Research Center which provides financial support through their Cooperative Agreement with the Minerals Management Service (MMS) and its Industry Consortium.

\section{References}

C. A. Brebbia. The Boundary Element Method for Engineers. Pentech Press, London, 1978.

J. W. Dold and D. H. Peregrine. Steep unsteady water waves : An efficient computational scheme. In proceedings 19th International Conference on Coastal Engineering, Houston, USA, pages 955-967, 1984.

O. M. Faltinsen. Numerical solution of transient nonlinear free-surface motion outside or inside moving bodies. Proceedings 2nd Int. Conf. on Num. Ship Hydrodynamics, U.C. Berkeley, pages 257-266, 1977.

J. D. Fenton. A fifth-order Stokes theory for steady waves. Journal of Waterways, Port, Coastal and Ocean Engineering, 111(2):216-234, 1985.

S. Grilli and I. A. Svendsen. An efficient boundary element method for nonlinear water waves. Engineering Analysis with Boundary Elements, 6(2):97-107, 1989.

S. T. Grilli and I. A. Svendsen. Corner problems and global accuracy in the boundary element solution of nonlinear wave flows. Engineering Analysis with Boundary Elements, 7(4):178-195, 1990.

M. A. Grosenbaugh and R. W. Yeung. Nonlinear free-surface flow at a twodimensional bow. Journal of Fluid Mechanics, 209:57-75, 1989.

S. A. Kinnas, Y.-H. Yu, B. K. Kacham, and H. S. Lee. A model of the flow around bilge keels of FPSO hull sections subject to roll motions. The 12th Offshore Symposium, Texas Section of SNAME,Houston, 2003.

W.-M. Lin. Nonlinear motions of the free surface near a moving body. Ph.D. Thesis, M.I.T., Dept. of Ocean Engineering, 1984.

W.-M. Lin, J. N. Newman, and D. K. Yue. Nonlinear forced motions of floating bodies. Proceedings 15th Symposium on Naval Hydrodynamics, Hamburg, 1984.

Y. Liu, M. Xue, and D. Yue. Computations of fully nonlinear three-dimensional wave-wave and wave-body interactions. Part II. Nonlinear waves and forces on a body. Journal of Fluid Mechanics, 438:41-66, 2001. 
M. S. Longuet-Higgins and E. D. Cokelet. The deformation of steep surface waves on water. I. A numerical method of computation. Proceedings of the Royal Society of London. Series A, Mathematical and Physical Sciences, 350(1660):1-26, July 301976.

W.H. Press, S. A. Teukolsky, W. T. Vetterling, and B. P. Flannery. Numerical Recipes in C, The Art of Scientific Computing. Cambridge University Press, 2nd edition, 1992.

H.G. Sung and S.T. Grilli. Numerical modeling of nonlinear surface waves caused by surface effect ships : Dynamics and kinematics. Proceedings 15th ISOPE Conference, Seoul, Korea, pages 124-131, 2005.

W. T. Tsai and D. K. P. Yue. Computation of nonlinear free-surface flow. Annual Review of Fluid Mechanics, 28:249-278, 1996.

T. Vinje and P. Brevig. Nonlinear ship motions. Proceedings 3nd Int. Conf. on Num. Ship Hydrodynamics,Paris, 1981.

L. C. Wrobel. The Boundary Element Method, VOLUME 1,Applications in ThermoFluids and Acoustics. Wiley, 2002.

R. W. Yeung. Numerical methods in free-surface flows. Annual Review of Fluid Mechanics, 14:395-442, 1982.

Y.-H. Yu, S. A. Kinnas, V. Vinayan, and B. K. Kacham. Modeling of flow around FPSO hull section subject to roll motion:Effect of the separated flow around bilge keels. Proceedings 15th Int. Offshore and Polar Eng. Conf., ISOPE, Seoul, Korea, 1:163-170, 2005. 